\title{
Spunti da una ricerca in corso. "La Rassegna. Pubblicazione Mensile della Camera di Commercio di California"
}

ISSN: 2282-4979

DOI: 10.12977/ere-

view116

Una selezione di alcuni documenti accompagna un testo che è il prodotto della prima disamina di una ricerca di dottorato attualmente in corso presso l'Università di Trieste. La complessa esperienza migratoria italiana negli Stati Uniti è stata analizzata dalla storiografia attraverso numerosi approcci metodologici, prendendo in esame un'ampia gamma di materiale archivistico. Attraverso alcune fonti selezionate dal Bollettino della Camera di Commercio Italiana in California negli anni Trenta del Novecento si vogliono ricostruire le dinamiche economico-commerciali dell'imprenditoria italoamericana in California, al centro di un ethnic business legato da un lato al mercato americano e dall'altro a quello dell'Italia fascista. La Camera di Commercio, attraverso la sua voce ufficiale, svela i nodi di una complessa rete transnazionale caratterizzata da intensi scambi di persone, capitali e merci tra le due Nazioni.

A selection of some documents will be followed by a contribution that is a first examination of a PhD research in progress at the University of Trieste. Scholars of the Italian migration in the United States adopted many methodological approaches, using a huge selection of documents from many archives. This contribution aims to depict the economic and commercial structures of the Italian entrepreneurs in California during the 30s. These entrepreneurs were at the center of an ethnic business that was strongly influenced by the American market and by the Italian fascist propaganda. The Italian Chamber of Commerce in California, through its official publication, "La Rassegna", reveals a transnational network characterized by a strong movement of people, goods and assets.

Tra la fine del XIX e l'inizio del XX secolo milioni di italiani si recarono negli Stati Uniti lasciando temporaneamente o definitivamente l'Italia. Una volta giunti a destinazione organizzarono la propria vita nelle grandi città all'interno di quartieri etnici, le cosiddette Little Italies, che riproducevano - e rielaboravano 
- le dinamiche sociali, culturali ed economiche che si erano lasciati alle spalle. Lingua, religione e alimentazione erano tre elementi che distinguevano immediatamente questi newcomers dagli altri gruppi presenti sul suolo americano. L'alimentazione in particolare fu uno dei sistemi attorno al quale si costruì l'identità etnica italoamericana e sul quale si coagularono importanti interessi economici. La ricerca mira alla ricostruzione delle dinamiche economico-sociali legate all'imprenditoria (produttori, esportatori, importatori) del settore alimentare italiano e italoamericano, nel periodo compreso tra il 1890 e il 1940. Uno studio che s'innesta su una prima considerazione riguardante la natura stessa della migrazione che va sempre analizzata sia come emigrazione che come immigrazione. Ciò significa che i migranti non tagliarono mai definitivamente i ponti con la madrepatria, ma mantennero legami che spesso erano funzionali alla definizione - e ridefinizione - della propria identità etnica [Waldinger 2016; Green, Waldinger (eds) 2016].

Le fonti attraverso le quali possono essere ricostruite queste reti transnazionali sono molteplici, si pensi alle lettere, che portavano informazioni e rimesse da una parte all'altra dell'Atlantico; ai quotidiani e alla produzione scritta dei movimenti politici - nello specifico quello anarchico presentò una grande capacità di legare aree geografiche in una fitta rete di relazioni tra Europa e America [Zimmer 2015]; o ai documenti delle istituzioni nate per organizzare i mercati, sotto la spinta dei crescenti interessi economici. In questo senso, rivestono una specifica importanza le Camere di commercio italiane all'estero, piattaforme economiche, politiche e sociali attraverso le quali i sistemi economico-produttivi possono scambiare merci, informazioni e capitali. Giovanni Luigi Fontana definisce storicamente le Camere come «entità bifronti»»»: non del tutto Stato e nemmeno completamente associazioni libere [Fontana 2001]. Una natura che le rende soggetti ibridi che «si prestano a servire da ponte tra lo Stato-nazione e la diaspora. Poiché appartengono sia all'uno che all'altra, esse gettano un ponte culturale, funzionale e strutturale tra l'Italia istituzionale e la rete della business community della diaspora» [Pozzi 2000, 262].

Espressione della classe dirigente locale, le Camere di commercio italiane negli Stati Uniti (Ccie) rappresentarono prevalentemente gli interessi di una classe sociale dapprima definibile come «borghesia dei servizi», solitamente di origine settentrionale, la quale perse progressivamente il ruolo di leadership quando an-

Fino allo scoppio della Grande Guerra l'emigrazione italiana fu caratterizzata dall'alta percentuale di ritorni. Per questo motivo chi migrava spesso non aveva interessi ad inserirsi nella comunità ospitante, tendendo dunque a conservare anche le proprie abitudini alimentari. 
che l'emigrazione meridionale riuscì a esprimere il proprio notabilato nella forma dei prominenti (una borghesia che aveva in mano gli interessi economici della comunità di appartenenza, come imprese di produzione, attività commerciali o carta stampata, e che spesso sfruttava la propria influenza per orientare i voti della comunità stessa verso un determinato partito politico o candidato). Queste dinamiche permisero alle Camere di divenire degli «agenti strutturanti» del flusso migratorio italiano, sviluppando «il loro ruolo di interfaccia della business community della diaspora» collegando «il contesto economico-politico locale e il sistema economico italiano» [Fontana 2001, 12]. L'analisi della composizione dei consigli camerali delle due più importanti Camere di Commercio italiane negli Stati Uniti (San Francisco e New York) rivela l'egemonia degli interessi legati al mercato alimentare, seguiti da quelli dell'abbigliamento.

A New York, Antonio Zucca - proprietario della Zucca and Co. Fruit Borkers and Commission Merchants, e presidente della Society of Italian Fruit Vendors - ricoprì il ruolo di presidente della Camera di Commercio dal 1899 al 1908, il periodo più lungo dei primi cinquant'anni di vita della Camera stessa (18871937). A San Francisco, il primo presidente della Camera - Giuseppe Onesti - era un commissionario di frutta e prodotti domestici. Grazie a una relazione di Carlo Dondero, redatta subito dopo la sua nomina a segretario della Camera nel 1885 e rivolta al Ministero degli Esteri italiano e al Ministero dell'agricoltura industria e commercio, si viene a conoscenza che nella città californiana erano attive circa 769 attività commerciali italiane di cui la maggior parte operavano nel mercato agroalimentare. Analizzare le Ccie statunitensi nel periodo compreso tra la fine del XIX secolo e la prima metà del XX secolo, significa studiare la complessa rete del mercato alimentare, dalla produzione al consumo² alla promozione commerciale di prodotti che ancora oggi sono uno dei simboli del made in Italy in tutto il mondo.

Nello specifico il contributo si propone uno sguardo sulla Camera di commercio di San Francisco negli anni Trenta e sull'utilizzo di un' importante fonte: "La Rassegna", bollettino ufficiale della Camera, attraverso il quale erano collegati gli interessi della business community italo-americana e quelli delle imprese (e degli enti statali) dell'Italia fascista. Durante questo periodo, e in particolare in occasione dell'attacco italiano all'Etiopia, si moltiplicarono le iniziative per incentivare l'acquisto di prodotti italiani negli Stati Uniti, per aiutare la penisola colpita dalle sanzioni della Società delle Nazioni [Luconi 2002]. Anche dopo la vittoria nel conflitto africano, continuò la mobilitazione e la propaganda di molte 
aziende sulle pagine dei quotidiani italo-americani, in particolare "Italian-Echo", "Il Popolo Italiano", "Gazzetta del Massachusetts" e "Il Progresso italo-americano" [Luconi 2005]. Questo aspetto mette in luce lo sforzo da parte del regime fascista di propagandare l'italianità, attraverso la promozione di scuole e la diffusione della cultura italiana grazie ad istituti preesistenti che furono fascistizzati - come la "Dante Alighieri" - e i Fasci italiani all'estero, ai quali fu «affidato il duplice incarico di promotori culturali ed economici, dovendo studiare forme di penetrazione economica e collaborazioni con l'Istituto nazionale di esportazione (Ine), creato nel 1926 per favorire l'esportazione di prodotti italiani e nel 1936 trasformato in Istituto nazionale fascista per il commercio estero» [Pretelli 2005, $524]^{3}$.

In questo contesto le Camere di commercio divennero vere e proprie casse di risonanza della propaganda del regime, come si può vedere dalle copertine di tre numeri apparsi nel 1936 nelle quali compaiono Mussolini, Vittorio Emanuele III e Rodolfo Graziani. Questa vicinanza al regime, però, comportò la temporanea chiusura di molte di esse all'indomani dell'ingresso in guerra dell'Italia fascista con la Germania di Hitler.

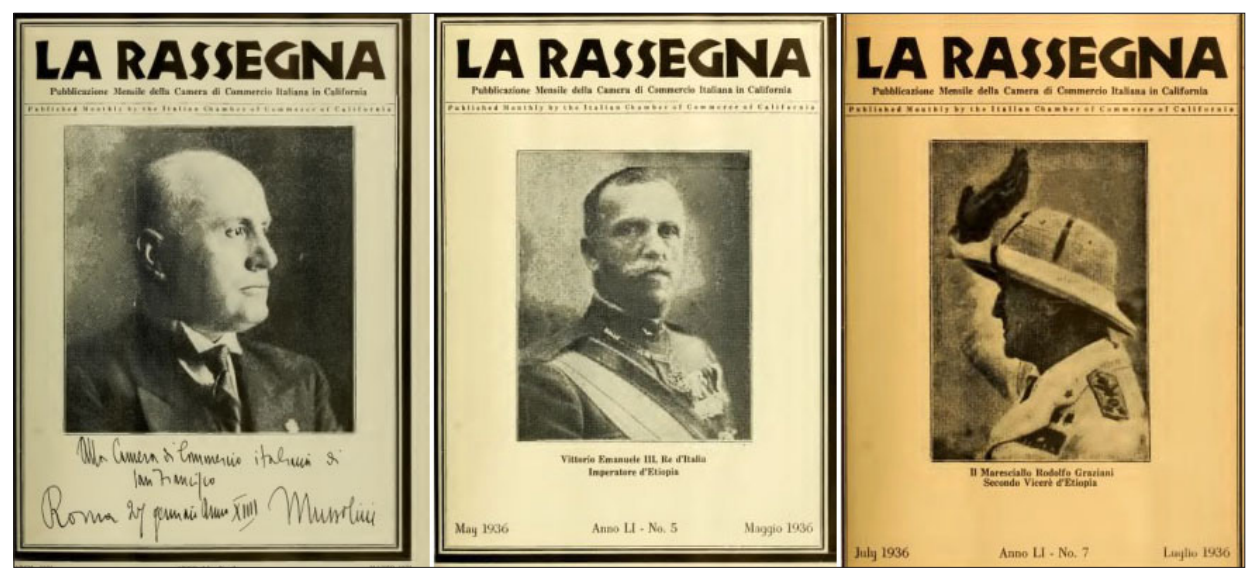

"La Rassegna", da sinistra a destra: marzo 1936, maggio 1936 e luglio 1936

L'ingerenza fascista in realtà bloccò in parte lo sviluppo libero delle Camere di Commercio. Come sostiene Pozzi:

La creazione di un ente ad hoc per l'intervento promozionale governativo all'estero - l'Istituto per il Commercio Estero nel 1926 - esprimeva le intenzioni accentratrici dell'intera politica del Regime verso la diaspora italiana: non una rete 
pluricefala e plurinodale, ma una pura e semplice protesi degli italiani d'Italia: non cittadini di Paesi esteri, ma italiani all'estero, da vincolare nuovamente alla madre Patria e soprattutto al Fascismo, trasformandoli in una sua presenza demoltiplicata sulla scena internazionale, e in uno strumento di influenza all'interno degli altri stati. In questa filosofia non c'era posto per l'associazionismo volontario che in qualche modo le Camere esprimevano [Pozzi 1993, 34].

La promozione dei prodotti italiani, o di "tipo italiano", cioè simili a quelli originali della Penisola, tendeva a rivolgersi da un lato a un mercato omogeneo - in cui i consumatori erano definiti "italiani", senza specificare le origini regionali - dall'altro ad un target cui si proponevano descrizioni che enfatizzavano le caratteristiche locali.

In the late 1920s, fascists began to acknowledge, extol, and commodify the very regional traditions and economies that Italian politicians had scorned among Italians in Italy and communities abroad just a decade before. Now, in their effort to promote commerce in Italian goods and a sense of national pride among Italians worldwide, fascists interacted with a flourishing immigrant cultural economy in which regionalism as an experience, language, and marketing strategy was already well established.

Advertisements in the Italian American press reveal an enduring dialectic between the local and the national, and between immigrants' everyday cultural and consumer identities and the diplomatic objectives of Italian political elites. This dynamic began at the turn of the twentieth century, as evidenced by publicity for exports in the Italian American press that advertised the regional origins of Italian products. During the late 1920s and 1930s, a transnational convergence occurred between Italian fascists, who crafted and exploited regional customs to advance a collective national identity within Italy, and immigrants in the United States who, while informed by the regional traditions of their local, paesi, increasingly conceived of themselves as sharing a common nationality. Promotional material for exports shows how tensions and alliances between the regional and the national manifested in both transnational market exchanges and in the quotidian experience of buying, selling, and consuming in ethnic enclaves [Zanoni 2014, 75].

La promozione dell'italianità era affidata anche a iniziative di carattere internazionale, come nel caso della New York's World Fair e la Golden Gate International Exposition di San Francisco, tenutesi entrambe nel 1939, per le quali, nonostante l'approssimarsi minaccioso della guerra, il regime investì ingenti energie e risorse [Carletti e Giometti 2016; Italy at the World's Fair, New York 1939 1940]. La Camera di commercio di San Francisco, inoltre, rappresentava ufficialmente l'Enit (Ente nazionale industrie turistiche) su tutta la costa pacifica, diventando così il punto di riferimento per le agenzie turistiche e per i privati, offrendo loro informazioni sulle possibilità di turismo in Italia.

Uno dei ruoli più importanti delle Ccie era collegare l'imprenditoria etnica con quella italiana. Per ricostruire questi legami è utile l'analisi del Bollettino degli 


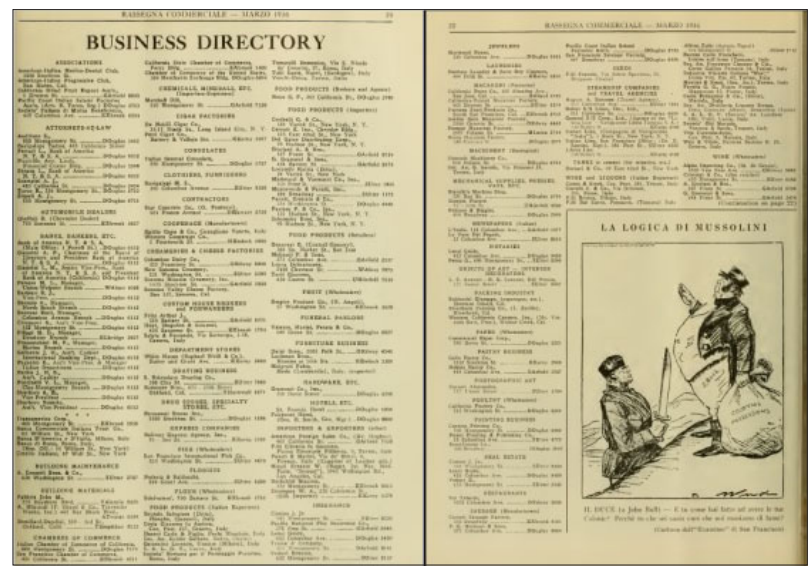

Business Directory, marzo 1936

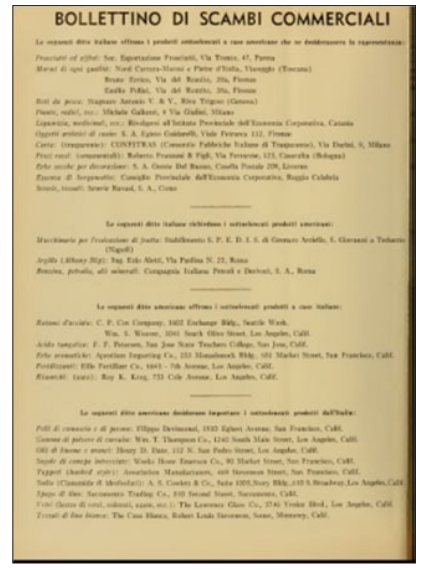

"Bollettino Scambi Commerciali”, marzo 1936

scambi commerciali e la Business directory, rubriche sempre presenti sulle pagine de "La Rassegna".

Come si può osservare, nella sezione denominata Business Directory sono presenti le aziende in affari con la comunità legata alla Camera di commercio, utile strumento per la conoscenza dei produttori e dei distributori di specifici prodotti ${ }^{4}$. La seconda sezione, invece, è una vera e propria bacheca di annunci nella quale da una parte le aziende italiane cercavano di introdurre il proprio prodotto negli Stati Uniti attraverso rappresentanti o agenti distributori, dall'altra le aziende americane - rappresentanti e distributori - cercavano prodotti italiani per i mercati locali. È il caso della Soc. Esportazione Prosciutti di Parma che pubblicò

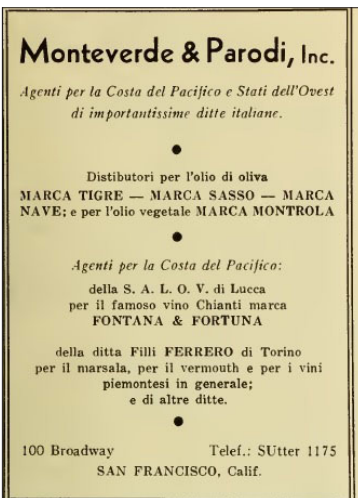

\section{G. GRANUCCI \& SONS}

Distributori esclusivi dei famosi prodoti "GOLD LABEL BRAND" -

Agenti per la Costa del Pacifico della ditta FRATELLI FOLONARI

grande Casa esportatrice di vini Chianti e di vini .

Distributori del sino Chianti marca

"Salov", della S. A. L. O. v. di Lucca.

Inoltre, distributori per altri tipi di vini italiani, francesi o tedeschi e per champagne e cogna rancesi.

424 Batroty Singer Telef:: GArfield 247 SAN FRANCISCO, Calif. un'inserzione per la vendita dei propri prodotti. I rappresentanti, inoltre, costituirono anche una fitta rete all'interno degli Stati Uniti, come testimoniato da alcune pubblicità.

La campagna Locatelli rivela che il mercato di San Francisco era collegato all'Italia anche attraverso la me-

Monteverde

Di questi si può notare la preminenza, negli anni Trenta, di produttori del nord Italia, tra cui la ditta Paolo Baratta di Parma. 
diazione del mercato di New York. Ettore Branchi, eletto nel 1935 segretario della Camera di Commercios, dipinse un quadro delle caratteristiche delle importazioni della costa pacifica compresi i problemi che dovevano affrontare produttori e distributori.

L'importazione italiana al

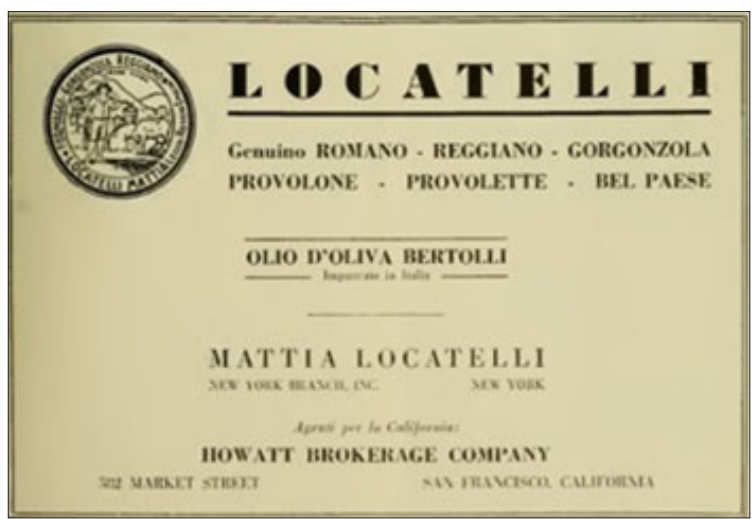

Locatelli

Pacifico è composta nella quasi totalità di prodotti alimentari. Si può dividere in diretta ed indiretta. La prima, segnalata dalle statistiche, proviene dall'Italia a mezzo delle motonavi della Compagnia "Libera Triestina" e della "Dollar Line". La seconda è effettuata dai grandi importatori di Nuova York e per via di terra raggiunge questi mercati. I porti di sbarco delle nostre merci sono quelli a cui fanno scalo le motonavi provenienti dall'Italia [...]. L'importazione dei prodotti alimentari sarebbe suscettibile di aumento se fosse organizzata in modo efficace e non lasciata alla sola iniziativa delle ditte locali. Con ciò non si vuol dire che i nostri importatori non facciano del loro meglio per acquistare i nostri prodotti, ma dato il loro legittimo interesse essi tendono a sostituire il prodotto italiano con altro prodotto quando i prezzi offerti si presentano inferiori. Per dare un esempio ricorderemo l'importazione dell'olio spagnolo che in fusti supera di gran lunga l'importazione similare italiana [Branchi 1935, 9-10].

L'opera di Branchi si concentrò sulla promozione della qualità del prodotto italiano contro le adulterazioni, in particolare dell'olio d'oliva', spesso in collaborazione con altre Ccie statunitensi; e su una campagna informativa riguardo i differenti metodi di packaging e di pagamento (assai diversi in Italia e Stati Uniti).

A mettere in collegamento i mercati, però, erano anche i produttori stessi che compivano numerosi viaggi per conoscere i consumi e i metodi di produzione americani. Il bresciano Antonio Folonari, proprietario di aziende agricole a Ludriano e nella zona bonificata di Ravenna, si recò nella metà degli anni Trenta ne-

Branchi era stato segretario della Camera di Commercio di New Orleans, a dimostrazione dei profondi legami che legavano tra loro le dirigenze italo-americane nelle grandi città degli Stati Uniti.

Lo scoppio della Guerra Civile Spagnola, però, avrebbe drasticamente ridotto le esportazioni di olio spagnolo, a vantaggio di quello italiano.

Importante fu la campagna congiunta che fecero le Ccie americane per la promozione del made In Italy. Cfr. Per la difesa dei Prodotti Italiani in America. Radio-discorso del Comm. Dott. Alberto Bonaschi 1936. 
gli Stati Uniti per un lungo tour toccando California, Michigan e New Jersey, per studiare i progressi nella coltivazione delle piante da frutta e nell'allevamento del bestiame [Cronache Camerali 1936]. Altrettanto facevano gli imprenditori italoamericani, che periodicamente si recavano in Italia per conoscere produttori e stipulare con essi contratti e accordi commerciali ${ }^{8}$. Queste dinamiche erano favorite dalle Ccie, che puntavano a creare collegamenti tra le due sponde, in particolare durante gli anni Trenta, quando il fascismo espresse un impegno maggiore per la promozione dell'italianità nelle comunità all'estero.

I documenti esaminati dimostrano la profonda interconnessione tra i mercati italiano e statunitense, rivelando lo scambio di informazioni, capitali e merci che hanno influenzato produzione e consumo di determinati prodotti. Il consumatore etnico stimolava certamente un certo tipo di mercato, che doveva poi però, essere organizzato. Processo che avvenne attorno a una classe di ethnic entrepreneurs [Waldinger, Aldrich, Ward 1990] che si posero come intermediari tra la produzione e il consumo servendosi e modellando il concetto di italianità al di fuori dei confini nazionali.

\section{Bibliografia}

Per la difesa dei Prodotti Italiani in America. Radio-discorso del Comm. Dott. Alberto Bonaschi 1936, «La Rassegna», febbraio.

Italy at the World's Fair, New York 1939 1940, Firenze: Vallecchi

Branchi E.C. 1935, Per una maggiore importazione dei prodotti Italiani sulla Costa del Pacifico, «Rassegna Commerciale», agosto

Carletti L. e Giometti C. 2016, Raffaello on the road. Rinascimento e propaganda fascista in America (1938-40), Roma: Carocci

Cinotto S. 2013, The Italian American Table. Food, Family, and Community in New York City, Urbana, Chicago and Springfield: University of Illinois Press

Fontana G.L. 2001, Per una storia delle Ccie e della business community italiana nel mondo, in Fontana G.L. e Franzina E. (eds), Profili di Camere di commercio italiane all'estero, vol. I, Unioncamere, Soveria Mannelli: Rubbettino

Green N. e Waldinger R. (eds) 2016, A Century of Transnationalism. Immigrants and their homeland connections, Urbana Chicago and Springfield University of Illinois Press

Lombino S. (ed) 2010, Avendo trovato l'America. Scritture di Viaggio tra Sicilia e nuovo

Si guardi in particolare la figura di Santo Garofalo, importatore di origine siciliana che a Chicago divenne presidente della Camera di commercio locale. Cfr. Lombino (ed.) 2010. 
mondo, Palermo: Fondazione Ignazio Buttitta

Luconi S. 2002, «Buy Italian». Commercio, consumi e identità italo-americana tra le due guerre, «Contemporanea», 3

Luconi S. 2005, Etnia e patriottismo nella pubblicità per gli italo-americani durante la guerra d'Etiopia, «Italia Contemporanea», 241

Pretelli M. 2005, Italia e Stati Uniti. "Diplomazia culturale” e relazioni commerciali dal fascismo al dopoguerra, «Italia contemporanea», 241

Pretelli M. 2012, La via fascista alla democrazia americana. Cultura e propaganda nelle comunità italo-americane, Viterbo: Sette città

Pozzi E. 1993, Verso la rete: l'esperienza storica del sistema delle camere italiane all'estero, «Impresa e Stato. Rivista della Camera di Commercio di Milano», 22

Pozzi E. 2000, Le Camere di commercio italiane all'estero nel secondo dopoguerra, in G. Sapelli (ed) 2000, Tra identità culturale e sviluppo di reti. Storia delle Camere di commercio italiane all'estero, Soveria Mannelli: Rubbettino

Waldinger R., Aldrich H. e Ward, R. 1990, Ethnic Entrepreneurs: Immigrant Business in Industrial Societies, Newbury Park London: Sage

Waldinger R. 2016, The Cross-Border Connection. Immigrants, Emigrants and their Homelands, Cambridge: Harvard University Press

Zanoni E. 2014, In Italy Everyone Enjoys It - Why Not in America?, Italian Americans and consumption in Transnational Perspective during the early Twentieth Century, in S. Cinotto (ed) 2014, Making Italian America. Consumer Culture and the Production of Ethnic Identities, New York: Fordham University Press

Zimmer K. 2015, Immigrants against the State. Yiddish and Italian Anarchism in America, Urbana, Chicago, and Springfield: University of Illinois Press 